\title{
Multilinguales
}

\section{Sur le classement des relatives adjectives}

On the Classification of Non-Restrictive Relative Clauses

\section{Jacques Evouna}

\section{(2) OpenEdition}

\section{Journals}

Édition électronique

URL : http://journals.openedition.org/multilinguales/1251

DOI : 10.4000/multilinguales. 1251

ISSN : 2335-1853

\section{Éditeur}

Université Abderrahmane Mira - Bejaia

\section{Édition imprimée}

Date de publication : 1 juin 2015

Pagination : 107-123

ISSN : 2335-1535

\section{Référence électronique}

Jacques Evouna, «Sur le classement des relatives adjectives », Multilinguales [En ligne], 5 | 2015, mis en ligne le 01 juin 2015, consulté le 17 septembre 2019. URL : http://journals.openedition.org/ multilinguales/1251 ; DOI : 10.4000/multilinguales.1251

Ce document a été généré automatiquement le 17 septembre 2019.

\section{(c) () (9)}

Multilinguales est mise à disposition selon les termes de la Licence Creative Commons Attribution Pas d'Utilisation Commerciale - Pas de Modification 4.0 International 


\section{Sur le classement des relatives adjectives}

On the Classification of Non-Restrictive Relative Clauses

Jacques Evouna

1 La question typologique dans l'analyse des subordonnées aboutit difficilement à un consensus chez les grammairiens, doublement fondée qu'elle est sur une définition approximative des critères traditionnels et sur une identification des faits elle-même peu rigoureuse. Ni la morphologie ni la syntaxe, ni le fonctionnement ni le sens n'ont été l'émanation d'une approche unanime et incontestable des structures subordonnées. Cela semble encore plus valable pour les relatives adjectives. Faut-il renoncer à toute tentative de classement de ces structures? On discute principalement d'un ensemble de faits fondateurs et caractéristiques des classements traditionnels des relatives adjectives, mais qui souvent, pour des raisons dont nous donnerons le détail, s'érigent rapidement en écueils.

Les questions abordées dans le cadre ainsi défini concernent la nature véritable des relatifs, l'élaboration du paradigme des relatives adnominales et la distinction de leurs valeurs d'emploi. En tout état de cause, nous procédons à une mise à jour de l'opposition radicale entre conjonction et pronom (1); nous restreignons la notion d'antécédent pour ériger en principe de classement l'unité fonctionnelle des relatives adnominales (2); nous tentons enfin d'affiner l'analyse des valeurs sémantiques des relatives adnominales (3).

\section{La forme des relatives adjectives}

2 La classe des relatifs est, à l'image de celle générale des pronoms, une sorte de fourretout, énumération pêle-mêle de classes syntaxiques et de catégories morphologiques que ne sous-tend visiblement aucune homogénéité formelle (Creissels, 1995 : 108), syntaxique ou même fonctionnelle. Selon K. Sandfeld (1928: xi) : «les mots qu'on est habitué à réunir sous le nom de pronoms sont de nature assez diverse ». En dépit du rôle de pronom qui leur est formellement reconnu, de nombreuses disparités émergent entre 
les relatifs. On affirme à ce sujet qu'« [ils] ne constituent pas une classe unitaire, mais des espèces différentes selon le mode de langage dont ils sont les signes » (Benveniste, $1966: 251$ ).

\section{La nature plurielle des relatifs}

3 Les disparités évoquées trouvent probablement leur origine dans l'inexistence d'une définition claire du terme "relatif ». Le Bon usage semble même déclarer la vanité de l'entreprise pour la classe générale des pronoms : «les noms ont une véritable définition, ce qui n'est pas le cas des pronoms» (Grevisse et Goosse, $2004: 625)$. C'est néanmoins au Bon usage que l'on doit aussi l'une des rares tentatives de définition du pronom relatif:

Les pronoms relatifs, qu'on appelle parfois conjonctifs, servent à introduire une proposition, qu'on appelle elle-même relative; à la différence des conjonctions de subordination (qui introduisent aussi une proposition), 1) ils ont une fonction dans cette proposition, celle de sujet, de complément, parfois d'attribut ; - 2) ils ont un genre, un nombre, une personne, même s'ils n'en portent visiblement pas les marques; - s'ils sont représentants, ils ont un antécédent. (2004 : § 677)

Cette définition, ainsi que les explications qui s'ensuivent, formule en réalité une série d'exceptions : «Qui, que, quoi peuvent être considérés comme des formes différentes, selon la fonction, du pronom qui "; "aucun des [pronoms de forme simple] ne varie en genre et en nombre». Cependant, "lorsqu'ils sont représentants, qui et que ont la personne grammaticale, le genre et le nombre de leur antécédent et transmettent cette personne, ce genre et ce nombre aux mots qui s'accordent avec eux ». Il est nécessaire, dans une telle situation de confusion, de réconcilier l'analyse (identification et typologie) des pronoms avec l'étymologie de ce terme. Pour Régnier-Desmarais,

Le mot de Pronom, en latin, porte en quelque sorte sa définition avec lui puisqu'il signifie qui tient lieu de nom, qui représente le nom, à peu près de même que Proconsul signifie qui représente le Consul, qui tient la place du Consul. (Cité par J. Pinchon, 1972:5)

5 Quels qu'ils soient, les éléments de la classe du pronom se définissent par leur capacité à se substituer au nom ou au substantif. Cela justifie l'appellation de "pronoms représentants ${ }^{1}$ », par opposition à des pronoms qui, eux, sont dits « nominaux ». En tout état de cause, les pronoms sont spécifiés par les catégories du genre et du nombre. Cependant, les formes engagées dans le processus de subordination sous l'étiquette de pronoms relatifs peuvent parfois perturber par une morphologie tantôt invariable, tantôt variable. Mais on préfère, en grammaire, les descriptions de relatifs de forme simple et de forme composée (Baylon et Fabre, 1978: 226; Grevisse et Goosse, 2004 : § 679). Les relatifs et les interrogatifs, la sous-classe des pronoms représentants varient, fait remarquer Le Bon usage, d'après leur fonction. Pourtant, « qui et que, lit-on en $\S 629$, transmettent le genre, le nombre et la personne de leur antécédent sans qu'ils portent eux-mêmes ces marques ».

6 Sont donc à prendre comme des relatifs invariables les formes " qui », «que ", " quoi », « où » et « dont ». En revanche, " lequel » et ses dérivés constituent le sous-paradigme des relatifs variables. A-t-on affaire, dans les deux cas, à des pronoms? Il semble bien qu'on assiste déjà tacitement, en grammaire traditionnelle, à la neutralisation de l'opposition morphologique entre conjonction et pronom ${ }^{2}$. Il s'agit cependant d'une situation de confusion qui dissimule assez grossièrement les difficultés des grammaires scolaires et même théoriques à identifier ces unités. Nous retiendrons ici deux critères d'érection des pronoms. Ainsi, et quoi qu'on dise, un pronom va demeurer un substitut 
du nom, c'est-à-dire une forme variable. Tout pronom est, comme le nom, précédé de l'indice de sa variabilité en une particule : l'article. Ces critères sommairement élaborés permettent de retenir «lequel » et ses dérivés comme les premiers pronoms relatifs. Quelle est donc la nature des autres?

7 Il est clair que le paradigme des relatifs rassemble des identités construites par fusion de différentes catégories. La fusion implique souvent des catégories grammaticales et énonciatives (Mod": Ass"/Inj") ${ }^{3}$. Il arrive aussi qu'elle associe des catégories grammaticales et des catégories fonctionnelles (qui) et/ou lexicales (lequel). À la base des relatifs se trouve soit la conjonction soit la subjonction, c'est-à-dire une particule modo-temporelle ou modale (Evouna, 2011b). Il est possible, désormais, de distinguer les relatifs selon que la modalité énonciative dont ils sont porteurs est spécifiée ou non par un mode verbal. Mais, quelque intéressante et séduisante que puisse paraître cette orientation, elle reste purement abstraite.

\section{Spécificités ou spécialisation fonctionnelles ?}

8 Comment expliquer la rection de certains par l'antécédent et l'insensibilité de certains autres aux marques d'accord (le genre et le nombre, en l'occurrence)? Si tous les relatifs remplissent un rôle syntaxique, qu'en est-il du rôle syntagmatique? Il est évident que les relatifs qui et que ne présentent pas les caractéristiques de substituts nominaux, ni même de nominaux. Une série de faits viennent mettre leur statut pronominal en doute. La formation de subordonnées relatives adjectives non standard fournit les tout premiers arguments de ce débat. On y voit invariablement apparaitre que, que la fonction engagée soit celle de sujet ou autre mais également une forme pronominale qui joue réellement ce rôle. Pourtant, selon les termes de Muller, on accorde en grammaire une fonction à certains relatifs. Qui et que, en l'occurrence sont présentés comme remplissant les postes fonctionnels de sujet et de complément d'objet direct (COD). Que ces formes soient, dans la classe des sujets et des COD, les seules exclues d'un ensemble d'opérations - la pronominalisation, la transformation passive ou la transformation emphatique - constitue plus qu'un motif d'interrogation.

9 Fournir en syntaxe la preuve que le qui dit sujet assume un terme de l'énoncé apparaît comme une démarche aussi difficile que coûteuse. Il déjoue, par exemple, toutes les règles d'accord du verbe. Quelle est, en outre, le fondement de la distinction sujet objet, étant donné qu'une telle spécialisation fonctionnelle ne peut être envisagée pour la classe des antécédents? Ce qui s'explique par les spécificités fonctionnelles dues probablement à l'occurrence d'une préposition dans la formation du relatif.

À la suite de Kayne (1975), il est confirmé que les relatifs «qui » et «que » sont des conjonctions exclues de tout rôle syntagmatique, que « dont » est un pronom adverbial relatif et que "où » est un adverbe relatif. La tradition grammaticale a occulté l'éventualité d'un rôle fonctionnel interne du support antécédent et «emberlificoté " l'explication de l'accord du verbe de tournures et exceptions confuses en érigeant une conjonction en sujet. Comment expliquer, autrement que par une exception, que qui et lequel soient des pronoms sujets à un même titre? Elle s'éloigne de la définition du pronom en y introduisant des éléments qui ne sont pas de même nature. En revanche, le statut de pronom est reconnu à une liste fermée d'unités constituée de «lequel » et de ses dérivés ${ }^{4}$. 
En outre, on parle bien, pour respecter une certaine tradition grammaticale, de conjonctives pour désigner des subordonnées qui commencent par une conjonction. Point de pronominales cependant, mais des relatives! Faute de pouvoir distinguer les différents opérateurs des relatives (conjonction relative, pronom relatif, adverbe relatif, pronom adverbial relatif), la grammaire entretient une situation confuse, puisqu'elle réserve indifféremment le statut de pronom à tous les relatifs. S'appuyant sur le principe de coréférence qui lie le relatif à son antécédent, Riegel et al. affirment : «[Le relatif] constitue un substitut du GN et assume une fonction dans la relative " (1994: 479). L'idée d'un pronom relatif invariable assurant la reprise d'un antécédent variable est plus que coûteuse.

Le statut et les fonctions que leur reconnaît la grammaire traditionnelle sur la base d'analogies purement distributionnelles sont en réalité ceux, soit de l'antécédent, soit des clitiques démonstratifs (ce, celui, celle, ceux, celles). Cela suggère, en conséquence, de retenir sur la liste des pronoms relatifs lequel et ses formes dérivées (lequel, laquelle, lesquels, lesquelles, auquel, à la laquelle, duquel, de laquelle, desquels, desquelles). Pour les autres formes, le statut qui convient le mieux est celui de conjonctions relatives (qui et que), d'adverbe relatif (où), de pronom adverbial relatif (dont) et de substantif relatif (quoi).

\section{Les relatives adjectives : des structures adnominales}

11 Avancer que les relatives adjectives appartiennent à un ensemble plus vaste, celui des relatives adnominales, est un rappel qui a sa pertinence. En outre, on peut se demander si le paradigme des relatives adjectives est l'unique pièce constitutive des relatives adnominales. À propos de structure, en effet, on semble a priori s'accorder sur le statut d'antécédent du nom support. Nous posons que la relative est une complétive dans le cas où le support n'est pas un antécédent.

\section{Les relatives complétives}

12 L'objectif de cette brève démonstration est d'inscrire sur la liste des relatives adjectives les structures relatives qui, pour la grammaire traditionnelle, remplissent la fonction dite de complément du nom. La logique de l'antécédence occulte la structure réelle des relatives adjectives. Plutôt que l'antécédent, il semble en effet plus productif de parler de sa nature nominale, le support nominal des relatives n'ayant pas toujours le statut d'antécédent. Il faut noter qu'il s'agit de structures à peu près ignorées. Une probable explication peut venir de la conception même de la relativisation, qui privilégie la coréférence à l'antécédent. Selon Muller (1996),

Un processus qui permet d'obtenir, à partir d'un constituant non verbal (antécédent), un constituant complexe comportant à sa tête ce constituant, et, en complément une proposition dans laquelle un des actants (au sens large: incluant les circonstanciels), est interprété comme coréférent à l'antécédent. (pp. 21-22)

13 Ainsi, et pour éviter tout malentendu, il importe, peut-être, de présenter succinctement le cas des subordonnées relatives en fonction de complément du nom (1) :

a. La salacité commence avec le regard de qui ne s'est pas accepté comme réserve de sensations inouïes et ineffables. (Exemple cité par le Bon usage)

b. Il n'est jamais du nombre de ceux qui forment un cercle pour discourir. (La Bruyère, Les Caractères ou les mours de ce siècle) 
L'une des indications à fournir ici c'est qu'il s'agit de structures qui mettent les grammaires scolaires devant une sorte de dilemme. Il y a par conséquent une certaine hésitation à les classer. La présence d'un nom support est facilement interprétée comme un cas d'antécédence. L'occurrence d'une préposition est, quant à elle, la manifestation incontestable du complément; raison pour laquelle elles sont identifiées comme des relatives substantives ${ }^{5}$. Une seule démarche peut aider à résoudre cette difficulté : la déconstruction de la phrase en deux énoncés (1') :

a. La salacité commence avec le regard de celui-ci. Celui-ci ne s'est pas accepté comme réserve de sensations inouies et ineffables.

b. Il n'est jamais du nombre de ceux-ci. Ceux-ci forment un cercle pour discourir.

Il apparaît que la chaîne de coréférence (Muller, 1996) lie deux positions plutôt que trois en situation d'antécédence: le relatif et la position vide en subordonnée. Autrement dit, le support nominal n'a pas le statut d'antécédent; et la préposition assure le lien entre le support et la subordonnée relative. Comme l'expliquent Riegel et al. (1994), et Grevisse (2004), le complément du nom est initié par une préposition.

On donne aux structures qui viennent d'être décrites le nom de «relatives complétives». La grammaire ne distingue-t-elle pas ailleurs les conjonctives complétives, mais au prix de l'application des mêmes critères de vacuité sémantique et d'absence de fonction syntagmatique de la conjonction ? Or, nous avons montré que qui et que, qui se retrouvent au premier plan ici, sont effectivement des conjonctions. Par "relatives complétives" donc, il faut simplement entendre "relatives» dont le support nominal n'est pas un antécédent et au sein desquelles le relatif n'assume pour toute fonction que celle de subordination. Évidemment, cette conception des « complétives » n'est pas définitive. Elle soulève d'autres questions, comme celle de savoir quelle différence faire désormais entre les «complétives conjonctives» et les « complétives relatives». Elle pourrait trouver ailleurs des terrains de réflexion.

\section{La fonction des relatives adnominales}

Lorsqu'on dit "relatives adjectives", c'est surtout en référence à une capacité fonctionnelle précise acquise par une structure. Ce fonctionnement de la subordonnée est généralement établi au moyen d'équivalences morphologiques et fonctionnelles. Ces équivalences trouvent leur fondement dans l'opération traditionnelle de substitution et leur explication théorique dans le principe structural de translation, soit pour Tesnière, l'opération qui "consiste à transférer un mot plein d'une catégorie grammaticale dans une autre catégorie grammaticale, c'est-à- dire à transformer une espèce de mot en une autre espèce de mot » (1959 : 364). La relative adjective est donc, de ce point de vue, une expansion du nom. Une sorte de débat, à ce sujet, oppose continuellement deux approches. Une certaine pratique dogmatique, qui reste d'ailleurs généralement sur l'appellation vague de "relatives avec antécédent », enseigne arbitrairement et de manière presque unanime que la relative se trouve en fonction de complément de l'antécédent (donc du nom). D'autres (Baylon et Fabre, 1978: 226 ; Riegel et al., 1994 : 480 ; Grevisse et Goosse, 2004: § 1059), par contre, analysent la relative adjective comme l'équivalent d'un adjectif qualificatif. Elle a donc la fonction " épithète ». Qu'en est-il exactement?

En outre, analyser une relative adjective comme un complément, c'est envisager, ce qui est absurde, la possibilité de reconnaître une fonction de complément de nom à un 
adjectif qualificatif. Enfin, quelques grammairiens soutiennent l'idée de «relatives adjectives » mises en apposition (Riegel et al., 1994). Cette conception de l'apposition est erronée. L'apposition est un processus qui établit entre les formes une égalité syntaxique. Elle a également des propriétés attributives. Or les tenants de cette analyse n'ont pas encore démontré l'égalité de la relative adjective et de son support, ni la possibilité qu'aurait une « relative adjective » d'assumer la fonction attribut.

Si nous poussons plus loin l'analyse, nous pouvons nous rendre compte que les "relatives complétives" sont un cas de translation double: le translatif (relatif) transfère la subordonnée dans la classe du nom ; le nom obtenu est à son tour transféré dans la classe de l'adjectif par la préposition. La translation autorise l'analyse du complément du nom comme une construction qui se retrouve dans le rôle syntagmatique d'épithète.

19 En conclusion, on restreint les capacités fonctionnelles des « relatives adjectives » à la seule fonction épithète non pas seulement de l'antécédent, mais également de l'éventuel support nominal. On a donc tort, comme le font les tenants des grammaires pédagogiques, de considérer exclusivement que les relatives adjectives sont des structures à antécédent. Il ressort en outre de la brève étude qui précède que le paradigme des expansions du nom illustre en profondeur une homologie fonctionnelle (Maingueneau, 1999: 79). Mais l'intérêt est d'avoir pu identifier une nouvelle catégorie des relatives adjectives : les « relatives complétives ».

\section{L'amalgame des valeurs cardinales}

20 La principale typologie sémantique en vigueur chez de nombreux auteurs ${ }^{6}$ émane en général de La Logique de Port-Royal. Cette grammaire avait défini deux valeurs pour les « relatives adjectives », distinguant ainsi, d'une part, les « relatives déterminatives » ou « restrictives» et, d'autre part, les « relatives explicatives». Chacune d'elles nécessite une brève explication pour démêler les valeurs rendues inextricables.

\section{La relative déterminative : une base relationnelle}

La définition de la "relative déterminative » est la même d'un auteur à un autre. Nous reprenons ici celle de Riegel et al. : «La relative est déterminative (ou restrictive) si elle est nécessaire à l'identification référentielle de l'antécédent, qu'il s'agisse d'un individu ou d'une classe, d'être réels ou virtuels» (1994: 484). Des questions se posent, cependant: les modalités et les valeurs de la relativisation d'un antécédent réel sont-elles les mêmes que celles de la relativisation d'un antécédent virtuel? en quoi consiste la détermination?

Nous n'avons pas l'intention de revenir ici sur les nombreux problèmes ${ }^{7}$ que soulève cette notion où se mêlent définition, identification, quantification, etc. Mais quelques précisions sont nécessaires. La détermination, d'où dérive l'adjectif "déterminatif ", renvoie à la valeur des déterminants (possessifs, démonstratifs, indéfinis, numéraux, etc.). Ces valeurs, en général, relèvent d'une relation abstraite ou concrète. Le déterminant établit et exprime entre son référent et un nom (le nom déterminé) une relation d'appartenance ou de position, une relation qualitative ou quantitative. Il existe pour chaque nom deux possibilités de détermination : l'une à gauche du nom et l'autre à sa droite. 

synonyme de "déterminative ». Il est important de proposer une explication à cette situation. Le sens même du mot « restriction » peut y aider. Lorsqu'en grammaire, par exemple, on parle de "subordonnée restrictive ", c'est en référence à l'expression d'une condition d'exception ou d'une expectative. En fait, la « relative restrictive » ne prédique pas, elle dit le monde tel qu'il est envisagé ou souhaité (3) :

a. Elles m'avaient mis si bien à mon aise, que ma langue parlait autant que mes yeux, quoiqu'elle ne dît pas les mêmes choses. (J-J. Rousseau, Confessions)

b. Il faut que tu guérisses, Électre, quel que soit le remède. (J. Giraudoux, Électre)

Quelle forme particulière rend possible l'expression linguistique de la condition d'exception? Le subjonctif n'y est pas totalement étranger. On le retrouve en effet dans les « relatives adjectives » (4):

a. Il évaluait le temps, et surtout l'heure du repas qui était la seule chose qui importât. (A.

Camus, La Peste) 
b. [...] Il semblait qu'il n'était pas un point de la ville qui ne fût placé dans la réverbération la plus aveuglante. (A. Camus, La Peste)

c. [...] En d'autres pays, on eût fait une chambre de justice qui eût consommé en frais le triple de l'argent volé, et qui n'eût rien remis dans les coffres du souverain.

(Voltaire, Zadig ou la destinée, histoire orientale)

\section{existence syntaxique autonome. La déconstruction des phrases permet de montrer} cette possibilité (5) :

a. Il évaluait le temps, et surtout l'heure du repas qui était la seule chose; que cette seule chose importât.

b. Il semblait qu'il n'était pas un point de la ville; que ce point de la ville ne fût placé dans la réverbération la plus aveuglante.

c. En d'autres pays, on eût fait une chambre de justice; que cette chambre de justice eût consommé en frais le triple de l'argent volé, et qu'elle n'eût rien remis dans les coffres du souverain.

En fait, les relatives restrictives sont introduites par la subjonction, forme homologue de la conjonction avec laquelle elle est souvent confondue. Mais les effets de la subjonction sont clairs : la dépendance de ces structures est davantage fonctionnelle que syntaxique ( $\mathrm{du}$ fait du lien lâche et défectible qui unit la subordonnée au support antécédent); leur valeur modale est injonctive. C'est en cela que se distinguent les « relatives descriptives ».

\section{La relative descriptive : le référent tel quel}

Un foisonnement de synonymes caractérise l'analyse des relatives adjectives. Ainsi, un terme apparaît souvent aux côtés des adjectifs « déterminative » et « restrictive ». C'est l'adjectif «descriptive ». Mais l'existence présumée d'une relation synonymique entre ces qualificatifs est désormais récusée. Nous avons vu que les relatives restrictives expriment tantôt une attente tantôt une restriction en relation avec un possible référent. À aucun moment, les relatives restrictives n'ont été envisagées comme relevant du processus de la prédication.

La relative sera dite « descriptive » si elle a un support (toujours un antécédent dans ce cas) réel et que son rôle consiste précisément à en donner une connaissance en vue d'une facile identification. Sa fonction, du point de vue littéraire, est alors documentaire, décorative ou symbolique (6):

a. [...] Et il cherchait le terme qui photographierait d'un seul coup la fastueuse jument qu'il imaginait. (A. Camus, La Peste)

b. Grand était le représentant réel de cette vertu tranquille qui animait les formations sanitaires. (A. Camus, La Peste)

33 À la différence des « relatives restrictives », les « relatives descriptives » emploient les temps du mode indicatif pour décrire, c'est- à-dire caractériser l'antécédent. La 
déconstruction des relatives descriptives montre que leur tête est une conjonction dont l'un des effets est l'établissement d'un lien fort ou indéfectible entre la subordonnée et son support antécédent : elles sont donc incapables d'avoir l'existence syntaxique d'une phrase. Autrement dit, les modalités de leur dépendance sont à la fois modales et fonctionnelles $\left(6^{\prime}\right)$ :

a. et il cherchait le terme; *que ce terme photographierait d'un seul coup la fastueuse jument; *qu'il imaginait cette jument fastueuse.

b. Grand était le représentant réel de cette vertu tranquille; que cette vertu tranquille animait les formations sanitaires.

Nous considérons la "relative adnominale » comme descriptive dès lors qu'elle dit le monde tel qu'il s'offre aux sens, tel qu'il est perçu. Les « relatives descriptives » sont des adjectifs qualificatifs de discours. On n'éprouvera donc pas de difficulté particulière à les remplacer par des adjectifs qualificatifs de langue. Quoi qu'il en soit, le principe de vériconditionnalité est applicable au contenu de ce type de relative. En d'autres termes, leur valeur modale assertive autorise à identifier comme vraies ou fausses les « relatives descriptives ».

\section{La relative explicative : une cause factuelle}

Un mot enfin sur les « relatives explicatives ». La définition qui en est proposée illustre parfaitement la lacune conceptuelle si symptomatique de la tradition grammaticale. Cette définition repose presque entièrement sur la négation des propriétés de la "relative déterminative». Riegel et al. fournissent la matière principale de cette nouvelle discussion :

La relative est explicative (ou appositive) lorsqu'elle ne joue aucun rôle d'identification dans l'identification référentielle de l'antécédent. Elle peut alors, sans dommage pour cette identification, être supprimée. (1994: 484)

Il faut tout de suite relever, quoi qu'on en dise, que ces structures n'ont presque rien à voir avec l'apposition, phénomène qui ne concerne du reste que certaines relatives substantives. Le seul détachement représente-t-il un argument suffisant pour déclarer une relative «appositive»? Les auteurs lient (p.480) l'appellation de «relatives adjectives » au fait qu'elles fonctionnent comme des adjectifs épithètes. Plus loin dans leur ouvrage (p.484), cependant, ils établissent une synonymie entre les adjectifs " explicative » et « appositive».

Pour revenir à la valeur d'explication, elle porte l'expression de cause narrative (7) :

a. La cour, qui donne le ton au reste de l'empire, était fort galante. (Diderot, Les bijoux indiscrets)

b. Une cousine à moi, qui sert de bonne dans la maison pauvre, range sur des planches rongées quelques assiettes de grosse faience. (J. Vallès, L'Enfant)

L'usage recommande, tant qu'il s'agit de "relatives adjectives", de concentrer son attention sur la relation avec l'antécédent. Comme la plupart des "adjectives», leur statut est celui de prédicat de l'antécédent. On se rend bien compte que les préoccupations syntaxiques et fonctionnelles prennent une fois encore le pas sur les valeurs que subsume l'appellation de «relatives explicatives ». Il convient, peut-être, de regarder ailleurs, en direction par exemple du procès dont l'antécédent est un des actants (toujours prime actant ici). C'est uniquement par rapport à ce procès que se justifient le sens et la valeur de la "relative explicative». Vue sous cet angle, elle se vêt 
des atours de cause factuelle ou positive. La fonction de la « relative explicative » n'est donc pas logique, mais narrative.

La "relative explicative ", comme du reste la "relative déterminative ", ne connait aucune spécialisation modo-temporelle. Elle emploie aussi bien les temps de l'indicatif que ceux du subjonctif. Comprise entre deux pauses brèves (virgules), elle entretient une relation logique implicite avec le procès auquel l'antécédent sert d'actant.

Au terme de la réflexion proposée ici, il apparaît que, sous quelque angle qu'elles soient considérées par la grammaire, les structures connues sous le nom de "relatives adjectives » posent essentiellement des problèmes de typologie, c'est-à-dire de délimitation du paradigme et d'identification de ses différents éléments constitutifs. Mais parler de "relative adjective ", c'est avoir une perception partielle du problème, car elles font partie des « relatives adnominales », classe dont elles représentent, selon toute probabilité, la pièce principale.

Mais il y a également les « relatives complétives » que l'on ne peut discerner au premier regard, à cause de certaines confusions et généralisations ou de l'emprise de l'intuition et du préfabriqué engendrés par les grammaires scolaires qui les classent dans les "substantives". Le statut de pronom est systématiquement appliqué à toutes les formes qui initient les « relatives adnominales».

Une analyse a néanmoins permis de montrer leur diversité morphologique. En plus des valeurs sémantiques habituelles et connues (déterminative et explicative), deux autres ont été formalisées (descriptive et restrictive), à partir de la déconstruction des relations synonymiques fragiles. Ce qui assez régulièrement est mis en cause, c'est la disparité ou le défaut de systématisation des critères traditionnels. Mais les critères traditionnels de classement s'avèrent contreproductifs principalement du fait de leur insuffisance conceptuelle.

\section{BIBLIOGRAPHIE}

BAYLON C. et FABRE P., Grammaire systématique de la langue française, Paris, Nathan, 1978.

BENVENISTE, E., Problèmes de linguistique générale, 1\&2, Paris, Gallimard, 1966, 1974.

CREISSELS D., Eléments de syntaxe générale I, Paris, PUF, 1995. DUBOIS J. et al., Dictionnaire de linguistique et des sciences du langage, Paris, Larousse, 1994.

EVOUNA J., « De la relativisation en français et en èwòndò : des particules énonciatives aux opérateurs syntaxiques ", in Studii de grammatica contrastiva, $n^{\circ} 16$, Editura Universitatii din Petesti, 2011a, pp. 29-43.

EVOUNA J., « L'a priori morphologique dans l'analyse des marqueurs de la subordination », in Mosaïques, « Les Idées reçues », Paris, L’Harmattan, 2011b, pp. 13-29.

EVOUNA J., L'Unité formelle des complétives. Esquisse de syntaxe catégorielle, Paris, Éditions des archives contemporaines, 2014.

GREVISSE M. et GoosSE A., Le Bon Usage, Bruxelles, De Boeck et Larcier, 14 ${ }^{\mathrm{ème}}$ édition, 2008. 
GREVISSE M. et Goosse, A., Le Bon usage, Bruxelles, De Boeck et Larcier, $13^{\text {ème }}$ édition, 2004.

KAYNE R. S., « French relative « que », part II », in Recherches linguistiques, 8, 1975, pp. 27-92.

MAINGUENEAU D., La Syntaxe du français, Paris, Hachette, 1999.

MULLER C., La subordination en français. Le schème corrélatif, Paris, Armand Colin, 1996.

MULLER C., « Sur les propriétés des relatives », in Cahiers de grammaire, 30, 2006, pp. 319-337.

PINCHON J., Les pronoms adverbiaux en et y. Problèmes généraux de la représentation pronominale, Genève, Droz, 1972.

REGNIER-DESMARAIS F.-S., ABBE François-Séraphin, Traité de la grammaire française, Paris, Coignard, 1706.

RIEGEL M., PELLAT J.-C. et RIOUL R., Grammaire méthodique du français, Paris, PUF, 1994.

SANDFELD K., Syntaxe du français contemporain. Les pronoms, Paris, Librairie ancienne H. Champion, 1928.

TESNIERE L., Éléments de syntaxe structurale, Paris, Klincksieck, 1959.

WILMET M., La Détermination nominale, Paris, PUF, 1986.

\section{NOTES}

1. C'est un point essentiel de la définition que propose le Dictionnaire de linguistique et des sciences du langage de J. Dubois (1994 : 408).

2. Clairement formulée par Muller (1996).

3. Analyse proposée par Evouna dans L'Unité formelle des complétives. Esquisse de syntaxe catégorielle, Paris, Editions des archives contemporaines, 2014.

4. Cf. Evouna, J., « De la relativisation en français et en èwòndò: des particules énonciatives aux opérateurs syntaxiques ", in Studii de grammatica contrastiva, $\mathrm{n}^{\circ}$ 16, Editura Universitatii din Petesti, 2011a, pp. 29-43.

5. C'est l'analyse qu'en fait Le Bon usage de Grevisse et Goosse (2004) ou la

6. Exemple de Tesnière (1959), de Baylon et Fabre (1978), de Grevisse et Goosse (2004) qui signalent deux principales subdivisions: les relatives déterminatives ou restrictives et les relatives explicatives.

7. Marc Wilmet leur a entièrement consacré son livre La Détermination nominale, Paris, PUF, 1986.

\section{RÉSUMÉS}

Nous examinons dans ce qui suit une question d'ordre typologique : le classement des relatives adjectives. La grammaire s'appuie, pour en établir les principales subdivisions, sur l'éventail de paramètres traditionnels, c'est-à-dire la morphologie, la syntaxe, le fonctionnement et le sens. Il semble pourtant qu'un défaut de cohésion caractérise continuellement la plupart des tentatives de cette entreprise de classement. Nous pensons que les relatives adjectives ne forment qu'un 
sous ensemble d'un paradigme plus vaste, celui des relatives adnominales dont la formulation actuelle des critères définitoires empêche de cerner l'unité profonde.

We examine in what follows a typological issue: the classification of restrictive and nonrestrictive relative clauses. The establishment of their main subdivisions leans on the traditional parameters: morphology, syntax, functioning, and meaning. However, it seems that a lack of cohesiveness regularly characterizes most attempts of this classification. It is thought that those relative clauses only form a subset of a wider paradigm, which is "adnominal relative clauses" whose current definition prevents from identifying the deep unit.

\section{INDEX}

Mots-clés : relative adjective, relative adnominale, relative complétive, déterminative, explicative, restrictive

Keywords : relative clause, adjective relative, adnominal relative, déterminative, explicative, restrictive

\section{AUTEUR}

\section{JACQUES EVOUNA}

ENS - Université de Maroua Maroua - Cameroun 